\title{
ТЕЗИСЫ ДОКЛАДОВ
}

\section{МЕЖДУНАРОДНАЯ КОНФЕРЕНЦИЯ}

«Физическая мезомеханика.

Материалы с многоуровневой иерархически организованной структурой и интеллектуальные производственные технологии",

посвященная 90-летию со дня рождения основателя и первого директора ИФПМ СО РАН академика Виктора Евгеньевича Панина

$$
\text { в рамках }
$$

Международного междисциплинарного симпозиума «Иерархические материалы: разработка и приложения для новых технологий и надежных конструкций»

5-9 октября 2020 года Томск, Россия 
Секция 4. Научные основы разработки материалов с многофазной иерархически организованной структурой, в том числе для экстремальных условий эксплуатации

\section{DOI: 10.17223/9785946219242/174 \\ ОПТИМИЗАЦИЯ ФУНКЦИОНАЛЬНЫХ СВОЙСТВ МОНОКРИСТАЛЛОВ ФЕРРОМАГНИТНЫХ СПЛАВОВ С ПАМЯТЬЮ ФОРМЫ ЗА СЧЕТ СТАБИЛИЗАЦИИ МАРТЕНСИТА НАПРЯЖЕНИЙ}

Панченко Е.Ю., Тимофеева Е.Е., Ларченкова Н.Г., Ефтифеева А.С., Тохметова А.Б.,

Тагильцев А.И., Суриков Н.Ю., Чумляков Ю.И.

НИ Томский государственный университет, Томск

В настоящей работе проведено систематическое исследование обратимой деформации в циклах нагрузка/разгрузка в зависимости от температуры испытания в состаренных под нагрузкой в мартенситном состоянии (СМH) монокристаллах сплавов $\mathrm{Ni}_{51} \mathrm{Fe}_{18} \mathrm{Ga}_{27} \mathrm{Co}_{4}$,

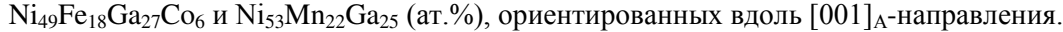

Для получения больших обратимых деформаций $\varepsilon_{\mathrm{rev}}$ до $13-15 \%$ под действием сжимающей нагрузки проводилась специальная СМН обработка монокристаллов - старение при $\mathrm{T}=423-498$ К, 1 ч в мартенсите, индуцированном сжимающими напряжениями, приложенными вдоль $[110]_{\mathrm{A}} \|[100]_{\mathrm{M}}[1,2]$. Такая СМН обработка во всех исследованных кристаллах приводит к стабилизации преимущественного раздвойникованного варианта мартенсита и появлению двустороннего эффекта памяти формы (ЭПФ) с обратимой растягивающей деформацией до 7-9 \% вдоль $[001]_{\mathrm{A}}$-направления и узкими температурными интервалами развития прямого $\Delta_{1}=\mathrm{M}_{\mathrm{s}}-\mathrm{M}_{\mathrm{f}}$ и обратного $\Delta_{2}=\mathrm{A}_{\mathrm{f}}-\mathrm{A}_{\mathrm{s}}\left(\Delta_{1}, \Delta_{2} \rightarrow 0\right)$ для $\mathrm{B} 2\left(\mathrm{~L} 2_{1}\right)-\mathrm{L} 1_{0}$ мартенситного превращения (МП) [1,2]. Впервые в ферромагнитных монокристаллах физическая причина стабилизации мартенсита за счет перераспределения атомов разного сорта и точечных дефектов в соответствии с симметрией $\mathrm{L}_{0}$-мартенсита была экспериментально подтверждена в работе [3] на примере монокристаллов сплава CoNiGa.

Изучение на монокристаллах исследуемых сплавов после СМН обратимых деформаций $\varepsilon_{\mathrm{rev}}$ вдоль $[001]_{\mathrm{A}}$-направления в циклах нагрузка/разгрузка при сжатии в широком температурном интервале от -70 до $+250^{\circ} \mathrm{C}$ позволило установить следующие общие закономерности их проявления. Во-первых, при температуре испытания $\mathrm{T}_{\mathrm{t}}>\mathrm{A}_{\mathrm{f}}$ монокристаллы проявляют сверхэластичность (СЭ) с обратимой деформацией $\varepsilon_{\mathrm{rev}}=3 \div 5 \%$ за счет развития прямых и обратных МП под нагрузкой (рис. 1). Кривые $\sigma(\varepsilon)$ характеризуются высокими критическими напряжениями $\sigma_{\mathrm{cr}}>100$ МПа и широким механическим гистерезисом $\Delta \sigma \geq 50$ МПа. В данном случае максимальная величина обратимой сжимающей деформации вдоль $[001]_{\mathrm{A}}$-направления $\varepsilon_{\text {rev }}$ близка к теоретической величине деформации $\mathrm{B} 2\left(\mathrm{~L} 2_{1}\right)-\mathrm{L} 1_{0}$ превращения в данных монокристаллах.

Во-вторых, при $\mathrm{T}_{\mathrm{t}}<\left(\mathrm{M}_{\mathrm{s}}+\mathrm{A}_{\mathrm{f}}\right) / 2$ в исследуемых монокристаллах вдоль $[001]_{\mathrm{A}^{-}}$ направления под действием сжимающей нагрузки наблюдаются большие обратимые деформации $\varepsilon_{\mathrm{rev}} \approx 13-15 \%$, которые почти в 3 раза превышают величину СЭ в этих образцах (рис. 1). В данном температурном интервале в образце при последующем охлаждении после $\mathrm{CMH}$ формируется стабилизированный вариант $\mathrm{L}_{0}$-мартенсита, который увеличивает размеры образца вдоль $[001]_{\mathrm{A}}$-направления. При достижении критических сжимающих напряжений $\sigma_{\mathrm{cr}}=10-30$ МПа деформация развивается за счет переориентации стабилизированного варианта $\mathrm{L}_{0}$-мартенсита в другой вариант мартенсита, возникающий под действием сжимающей нагрузки. При этом заданная деформация может быть полностью обратима в циклах нагрузка/разгрузка, т.е. наблюдается ферроэластичность (ФЭ) с $\varepsilon_{\mathrm{rev}} \approx 13-$ $15 \%$ во всем температурном интервале при $\mathrm{T}_{\mathrm{t}}<\left(\mathrm{M}_{\mathrm{s}}+\mathrm{A}_{\mathrm{f}}\right) / 2$. Если деформирующие напряжения для переориентации вариантов мартенсита ниже, чем величина механического гистерезиса $\Delta \sigma$, то заданная деформация до $13-15 \%$ является обратимой только после снятия нагрузки и небольшого перегрева, т.е. наблюдается ЭПФ с $\varepsilon_{\mathrm{rev}} \approx 13-15$ \% (рис.1).

B-третьих, существует узкий температурный интервал $\left(20-30^{\circ} \mathrm{C}\right)$ при $\left(\mathrm{M}_{\mathrm{s}}+\mathrm{A}_{\mathrm{f}}\right) / 2<$ $\mathrm{T}_{\mathrm{t}}<\mathrm{A}_{\mathrm{f}}$, в котором наблюдаются двухстадийные кривые $\sigma(\varepsilon)$. Первая стадия на кривых $\sigma(\varepsilon)$ характеризуется низкими критическими напряжениями $\sigma_{\mathrm{cr}}<5 \div 15$ МПа и деформацией 8-9\%, которая является обратимой при снятии нагрузки и изменении температуры. Вторая стадия 
Секция 4. Научные основы разработки материалов с многофазной иерархически организованной структурой, в том числе для экстремальных условий эксплуатации

на кривых $\sigma(\varepsilon)$ представляет собой кривую СЭ с высокими значениями $\sigma_{\mathrm{cr}}>100 \mathrm{MПа} \mathrm{и} \Delta \sigma$ $\geq 50$ МПа. Суммарная обратимая деформация на двух стадиях достигает $13-15 \%$.

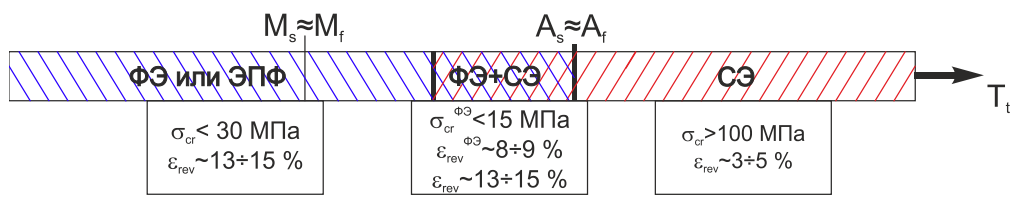

Рис. 1. Схема, демонстрирующая температурные интервалы проявления функциональных свойств в циклах нагрузка/разгрузка при сжатии в монокристаллах сплавов $\mathrm{Ni}_{51} \mathrm{Fe}_{18} \mathrm{Ga}_{27} \mathrm{Co}_{4}, \mathrm{Ni}_{49} \mathrm{Fe}_{18} \mathrm{Ga}_{27} \mathrm{Co}_{6}$ и $\mathrm{Ni}_{53} \mathrm{Mn}_{22} \mathrm{Ga}_{25}$, ориентированных вдоль [001] после СМН при $\mathrm{T}=423-498 \mathrm{~K}$ в мартенсите, индуцированном сжимающими напряжениями вдоль $[110]_{\mathrm{A}} \|[100]_{\mathrm{M}}$.

Исследование калорических эффектов в данном интервале температур показало, что на первой стадии при нагрузке наблюдается обратный калорический эффект - поглощение тепла, что соответствует обратному МП. Далее на второй стадии обнаружено выделение тепла при нагрузке, и поглощение тепла при разгрузке, что соответствует развитию прямого и обратного МП при проявлении СЭ. Поэтому предполагается, что под действием сжимающей нагрузки вдоль [001] $]_{\mathrm{A}}$-направления вблизи температур обратного МП $\mathrm{A}_{\mathrm{s}} \approx \mathrm{A}_{\mathrm{f}}$ в исследуемых кристаллах сначала наблюдается обратное МП стабилизированного варианта $\mathrm{L} 1_{0}$-мартенсита в аустенит при низких критических напряжениях. Затем, при увеличении деформирующих напряжений имеет место развитие МП под нагрузкой с образованием другого варианта мартенсита, который характеризуется сжимающей деформацией вдоль $[001]_{\mathrm{A}}$-направления.

Таким образом, экспериментально показано, что за счет старения при $\mathrm{T}=423-498 \mathrm{~K}$ в мартенсите, индуцированном сжимающими напряжениями вдоль $[110]_{\mathrm{A}} \|[100]_{\mathrm{M}}$, можно разрабатывать монокристаллы ферромагнитных сплавов с уникальными функциональными

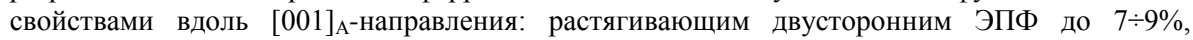
большими обратимыми деформациями до $13-15 \%$ при сжатии с низкими критическими напряжениями $\sigma_{\mathrm{cr}}<30 \mathrm{MПа}$ в широком температурном интервале и обратным эластокалорическим эффектом (поглощением тепла при нагрузке) при действии низких деформирующих напряжений $\sigma_{\mathrm{cr}}<5 \div 15 \mathrm{MПа}$. Такие материалы могут найти широкое практическое применения в различных современных технологиях в качестве рабочих тел термо- и магнитоуправляемых силовых приводов, датчиков, твердотельных холодильников, тепловых насосов и демпфирующих устройств.

Исследование выполнено за счет гранта Российского научного фонда № 20-19-00153.

1. Panchenko E., Timofeeva E., Eftifeeva A, et al. Giant rubber-like behavior induced by martensite aging in $\mathrm{Ni}_{51} \mathrm{Fe}_{18} \mathrm{Ga}_{27} \mathrm{Co}_{4}$ single crystals // Scripta Mater. 2019. V.162. P.387-390.

2. E. Panchenko, E. Timofeeva, M. Pichkaleva, A. Tokhmetova, N. Surikov, A. Tagiltsev, Y. Chumlyakov. Effect of Stress-Induced Martensite Aging on Martensite Variant Reorientation Strain in NiMnGa Single Crystals// Shape Memory and Superelasticity. 2020. V.6. P. 29-34.

3. Kadletz P., Krooß P., Chumlyakov Yu., et al. Martensite stabilization in shape memory alloys - Experimental evidence for short-range ordering // Materials Letters. 2015. V. 159. C.16-19. 